Document downloaded from:

http://hdl.handle.net/10251/171686

This paper must be cited as:

Vallejo-Castro, L.; Ortega Tamarit, B.; Bohata, J.; Zvanovec, S.; Almenar Terre, V. (2020). Photonic multiple millimeter wave signal generation and distribution overreconfigurable hybrid SSMF/FSO links. Optical Fiber Technology. 54:1-7.

https://doi.org/10.1016/j.yofte.2019.102085

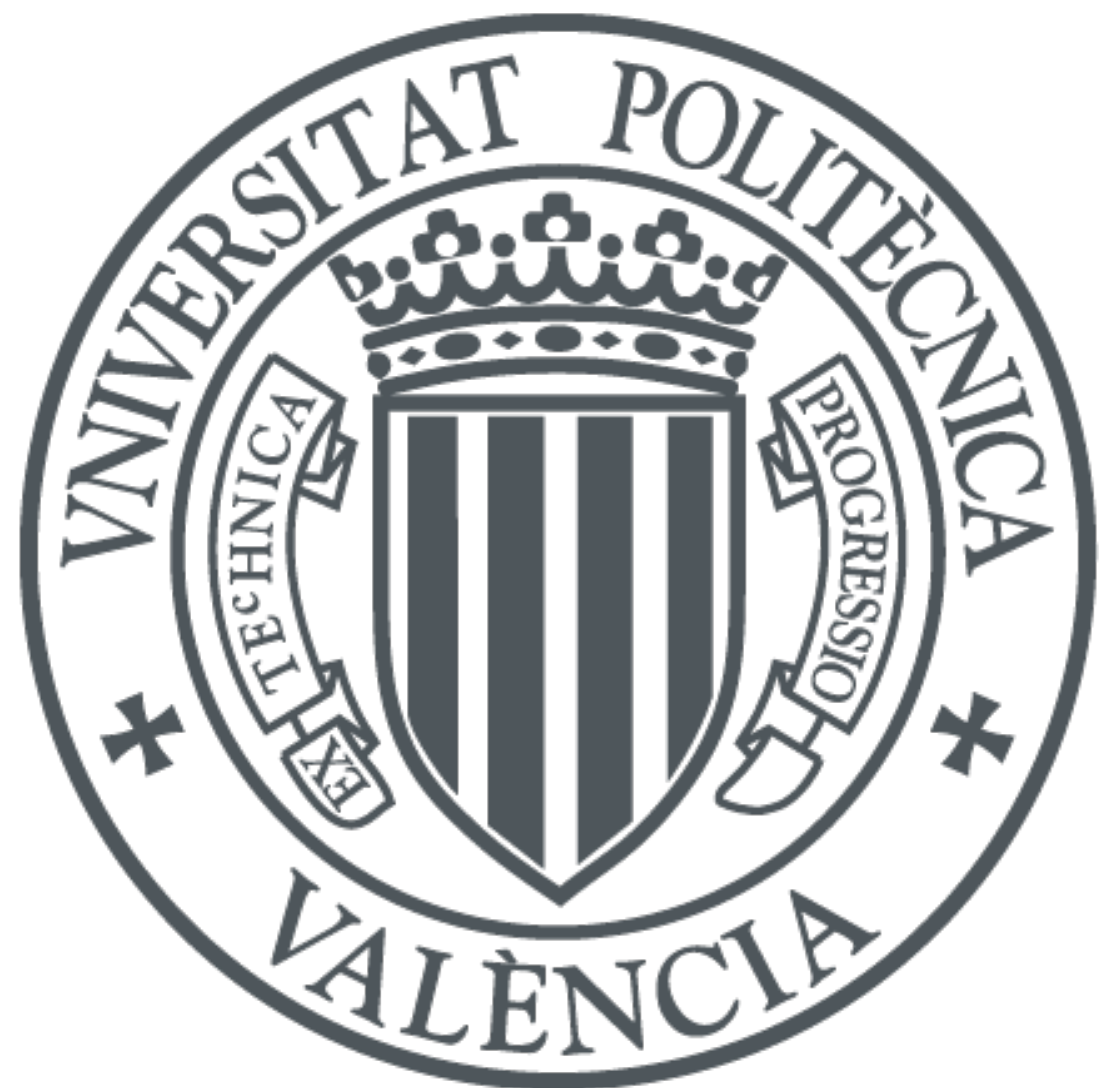

The final publication is available at

https://doi.org/10.1016/j.yofte.2019.102085

Copyright Elsevier

Additional Information 


\title{
Photonic multiple millimeter wave signal generation and distribution over reconfigurable hybrid SSMF/FSO links
}

\author{
Luis Vallejo, ${ }^{1,}{ }^{*}$ Beatriz Ortega, ${ }^{1}$ Jan Bohata, ${ }^{2}$ Stanislav \\ ZVANOVEC, $^{2}$ VICEnÇ Almenar ${ }^{1}$
}

${ }^{1}$ Instituto de Telecomunicaciones y Aplicaciones Multimedia, Universitat Politècnica de València, Camino de vera, s/n 46022 Valencia (Spain)

${ }^{2}$ Department of Electromagnetic Field, Faculty of Electrical Engineering, Czech Technical University in Prague, Technicka 2, 16627 Prague (Czech Republic),

*Corresponding author:luivalc2@iteam.upv.es

\begin{abstract}
Microwave photonics provides attractive solutions for millimeter wave (mmW) signal generation. In this paper, we demonstrate photonically generated multiple $\mathrm{mmW}$ signals transmission over a wavelength division multiplexed (WDM) hybrid optical network based on optical fiber and free-space optics (FSO) links. The experimental results demonstrate the generation and reconfigurable signal distribution from a central office to base stations in the frequency range $14-40 \mathrm{GHz}$ with phase noise levels below $-87 \mathrm{dBc} / \mathrm{Hz}$. Moreover, $10 \mathrm{~Gb} / \mathrm{s}$ data transmission has been demonstrated over photonically generated $40 \mathrm{GHz} \mathrm{mmW}$ signal. We show that FSO technology provides a possible solution for $\mathrm{mmW}$ fronthaul in 5th generation networks to extend the optical access network providing increased wireless accessibility and maintaining transmission capacity.
\end{abstract}

Keywords: Microwave photonics, millimeter-waves, external modulation, free-space optics, wavelength division multiplexing.

\section{Introduction}

The next 5th generation $(5 \mathrm{G})$ and beyond wireless networks are aimed to provide unprecedented data rates to mobile users by implementing modern technologies enabling a number of networking challenges such as high capacity and mobility, large coverage and low latency [1].

The incorporation of millimeter wave $(\mathrm{mmW})$ signals with the large available spectrum, up to $110 \mathrm{GHz}$ and beyond [2], allows efficient frequency reuse in small cell network architectures, minimal interference and promising higher data rates and traffic. As it is wellknown, photonics plays a key role in mobile networks and moreover, it also can enable effective transportation of radio signals over large distances. For example, radio-over-fiber (RoF) systems operating at $\mathrm{mmW}$ have been subject to extensive research due to their advantages such as low attenuation, transparency to modulation formats, high capacity, flexibility and dynamic resource allocation [3]. Therefore, the RoF technology can be a possible solution for the cloud radio access network (C-RAN) [4]. In these systems, an optical distribution network $(\mathrm{ODN})$ delivers $\mathrm{mmW}$ signals from a central station (CS) containing data resources and optical transmitters and receivers to many remote base stations (BS), which merely consist of an optical to electrical converter, electrical amplifiers and antennas $[5,6]$.

The overall performance assessment of such RoF system has to be optimized according to the figures of merit of the different subsystems with regards to the bandwidth, transmission integrity, scalability, reliability, availability, power consumption, costs and simplicity requirements [7]. However, one of the most challenging stages in these systems represents the generation of the $\mathrm{mmW}$ signals due to the limited frequency response of electronic components.

Microwave photonic solutions for $\mathrm{mmW}$ signal generation have been extensively studied in the literature where stable and flexible signals have been demonstrated by employing a 
variety of techniques [8]. The optical beating of two heterodyne lasers with emission frequencies separated by the desired frequency allows generating signals in a broad frequency range. However, either an automatic phase control loop in uncorrelated lasers or an optical sideband injection locking in correlated lasers schemes is required for frequency and phase stabilization $[9,10]$. Multimode light sources, such as dual-mode lasers, mode-locked lasers, Fabry-Perot lasers or supercontinuum sources have also attracted attention as low-cost alternatives to obtain different beating tones for $\mathrm{mmW}$ signal generation offering scalability to several wavelength division multiplexing (WDM) channels, however with customized designs required [11,12]. Other techniques employing optical fiber nonlinear mechanisms such as fourwave mixing [13], cross gain modulation [14] or stimulated Brillouin scattering (SBS) [15] have also been explored for $\mathrm{mmW}$ signal generation even though cost and complexity of these setups increase hugely.

Moreover, the external modulation of a continuous wave $(\mathrm{CW})$ laser by an intermediate frequency allows generating a $\mathrm{mmW}$ signal when two sidebands, separated by the desired frequency, are selected, and therefore, optical frequency multiplication (OFM) is held at the electro-optical conversion [16]. OFM has been proposed either by the generation of higherorder harmonics or also by the suppression of the optical carrier allowing the use of multiple Mach-Zehnder modulators (MZM) to increment the order of the OFM. Frequency doubling, quadrupling and up to octupling [17] have been recently demonstrated with simplicity and in particular with reduced electronic bandwidth requirements for $\mathrm{mmW}$ signals generation as the main advantage. Furthermore, multiband signal transmission based on RoF technology has been demonstrated as an attractive choice according to the standards to carry single or multiple services in baseband, microwave and $\mathrm{mmW}$ bands [18].

In addition, the free-space optics (FSO) communication has been identified as a promising solution for $5 \mathrm{G}$ and optical access networks extension where spatial and WDM can be adapted to meet future capacity and coverage challenges [19]. Indeed, hybrid $\mathrm{mmW}$ and FSO architectures were demonstrated using an adaptive diversity combining technique for mobile backhaul transportation. For example, $60 \mathrm{GHz}$ fiber-wireless integrated mobile backhaul networks have been demonstrated using adaptive techniques at the receiver side based on a maximum ratio combining algorithm to compensate losses by fog and turbulence [20]. In [21], a novel coordinated mapping and combining technique was employed and experimentally verified to increase the transport reliability of such next-generation mobile networks by sensing the signal quality in each data block and adapting the load accordingly. Recently, the FSO channel has also been deployed in radio over FSO (RoFSO) system proposed for $5 \mathrm{G}$ networks and the system performance has been evaluated under the presence of atmospheric turbulence [22].

In this paper, we propose and experimentally demonstrate multiple $\mathrm{mmW}$ signals photonic generation and distribution over a WDM hybrid network for increased reconfigurability and flexibility, and also leading to dynamic resources and services allocation in future networks. The signals are distributed through a hybrid network comprising an optical fiber and FSO link where optical channel selection is provided at the remote node $(\mathrm{RN})$ by an arrayed waveguide grating (AWG) to optically route the desired $\mathrm{mmW}$ frequency towards the corresponding BS with the very low phase noise level. Finally, $10 \mathrm{~Gb} / \mathrm{s}$ data signal transmission with 32quadrature amplitude modulation (QAM) is successfully demonstrated over $40 \mathrm{GHz} \mathrm{mmW}$ signal along the hybrid link with minimum EVM of $8 \%$.

\section{Principle and experimental setup}

The photonic multiple $\mathrm{mmW}$ signal generation and distribution system is based on a number of optical carrier signals in the WDM scheme, as depicted in a network example in Fig. 1(a), where each optical channel carries one of the $\mathrm{mmW}$ signals to be transmitted through the hybrid 
network. In this architecture, each optical wavelength is modulated at the CS by an intermediate frequency $\left(f_{R F}\right)$, using an external MZM with bias voltage:

$$
V(t)=V_{D C}+V_{R F} \cos \left(w_{R F} t\right)
$$

where $V_{D C}$ is the bias DC voltage, and $V_{R F}$ and $w_{R F}$ are the amplitude and angular frequency of the sinusoidal signal. As it is well known, the output optical field of the MZM can be written as:

$$
E_{\text {out }}(t)=E_{0} \cos \left(\frac{\pi V_{D C}}{2 V_{\pi}}+\frac{\pi V_{R F}}{2 V_{\pi}} \cos \left(w_{R F} t\right)\right) \cos \left(w_{c} t\right)
$$

where $E_{0}$ and $w_{c}$ are the amplitude and angular frequency of the input optical carrier, respectively and $V_{\pi}$ is the half-wave modulator voltage.

Provided $V_{D C}=V_{\pi}$, and using Bessel functions, eq. (2) can be rewritten as [23]:

$$
\left.E_{\text {out }}(t)=E_{0}\left\{J_{1}\left(\frac{\pi V_{R F}}{2 V_{\pi}}\right) \cos \left(\left(w_{c}+w_{R F}\right) t\right)+J_{1}\left(\frac{\pi V_{R F}}{2 V_{\pi}}\right) \cos \left(\left(w_{c}-w_{R F}\right) t\right)\right)\right\}
$$

where the optical carrier is suppressed without a need for optical filters.

By beating both sidebands at the optical receiver in the BS, the generated $\mathrm{mmW}$ signal is double the drive frequency, $f_{m m W}=2 f_{R F}$. Using this approach brings another advantage, besides the reduced electronic bandwidth requirements at the transmitter, which is the robustness of the system to fiber dispersion-induced RF power penalties [24]. However, a relatively high RF drive power is required to obtain a desirable modulation depth due to the nonlinear bias point [6]. Different optical signals are multiplexed at the CS and distributed along the optical network, which includes combined optical fiber and FSO links. The usage of FSO technology in 5G networks allows providing an effective solution for $\mathrm{mmW}$ fronthauling to extend the optical access network, i.e., to inaccessible or restricted areas for fiber installations, or to provide extended wireless accessibility by exploiting the FSO signal propagation advantages compared to $\mathrm{mmW}$ signal limitations [25].

At the RN, the optical signals are distributed by a demultiplexer, and each optical channel is routed towards the corresponding BS, where opto-electrical conversion is held at the photodetector, and the $\mathrm{mmW}$ signal at the target frequency is amplified and radiated by an antenna. Higher network capacity can be then directly obtained by increasing the number of WDM channels. 


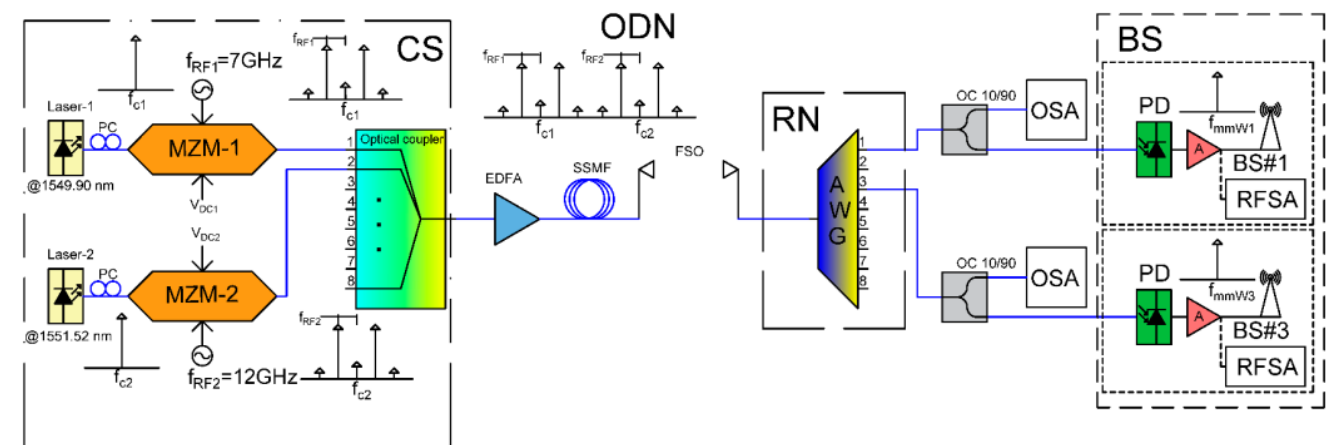

(a)

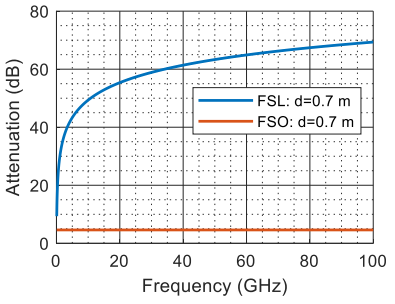

(b)

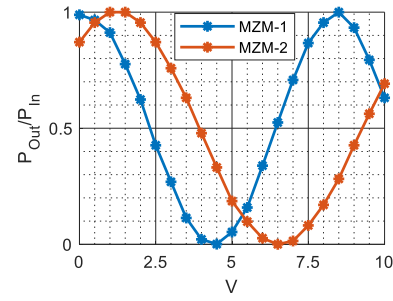

(c)

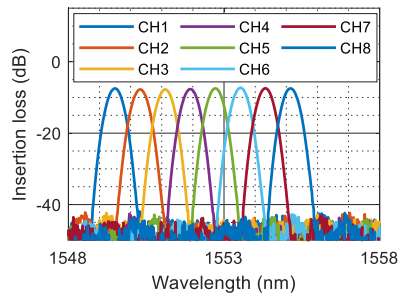

(d)

Fig. 1. Principle of multiple mmW signal generation and distribution through an hybrid SSMF/FSO system: (a) Schematic layout (CS: central station, PC: polarization controller, MZM: Mach-Zehnder modulator, ODN: optical distribution network, EDFA: erbium-doped fiber amplifier, SSMF: standard single mode fiber, FSO: free-space optics, RN: remote node, AWG: arrayed waveguide gratings, OC: optical coupler, OSA: optical spectrum analyzer, BS: base station, PD: photodetector, A: RF amplifier, RFSA: RF spectrum analyzer), (b) Losses for radio FSL (free-space loss) and FSO (free space optics), (c) Measured output/input optical power transfer function of an MZM as a function of the bias voltage $(V)$, (d) Output spectral response of 8 channel AWG with $100 \mathrm{GHz}$ channel spacing.

In our experimental setup, two distributed feedback (DFB) lasers (EXFO IQS) (CW Laser1 and Laser-2) emitted continuous wave (CW) optical carrier signals with $10 \mathrm{dBm}$ output power at $1549.90 \mathrm{~nm}$ and $1551.52 \mathrm{~nm}$ wavelengths. After the optimization of the polarization state of the input signal by a polarization controller (PC), the first signal was modulated by a single drive MZM-1 (Avanex PowerBit F-10), and the second one employed MZM-2 (Sumitomo Osaka Cement T DEH1.5-40). Fig. 1(c) shows the experimental power-voltage curve of both MZMs, where the zero bias point was determined as $4.5 \mathrm{~V}$ and $6.5 \mathrm{~V}$ for MZM1 and MZM-2, respectively. The RF driving signals were generated by signal generators (Agilent PNA-X N4373C and PSG E8267C) at frequencies of $7 \mathrm{GHz}$ and $12 \mathrm{GHz}$ with $18 \mathrm{dBm}$ and $23 \mathrm{dBm}$ output power, respectively.

The optical modulated signals were multiplexed by an optical coupler and afterward amplified by an erbium-doped fiber amplifier (EDFA) (Amonics EDFA-23-B-FA) with $13 \mathrm{dBm}$ output power to be transmitted by $10 \mathrm{~km}$ standard single mode fiber (SSMF) and then launched into the $0.7 \mathrm{~m}$ long FSO system by using graded-index pigtailed lenses (GRIN, Thorlabs 50-1550A-APC) with an aperture of $1.8 \mathrm{~mm}$, and plano-convex lenses with a diameter of $25.4 \mathrm{~mm}$. The optical beam was collimated at the receiver by the combination of the same lenses. Fig. 1(b) shows the significant frequency independence of losses for $0.7 \mathrm{~m}$ long FSO link in our experimental setup (i.e. flat $4.6 \mathrm{~dB}$ loss), compared to RF free space loss (FSL), which is exponentially frequency dependent.

At the RN, the signal was launched into a $1 \times 8$ AWG with a free spectrum range (FSR) of $100 \mathrm{GHz}(0.8 \mathrm{~nm})$, as shown in Fig. 1(d), where optical channels came out from output ports 
$\# 1$ and \#3, respectively. The AWG output ports were connected to a 90/10 optical coupler allowing the monitoring of the signal by the optical spectrum analyzer (OSA), whereas the RF double-frequency signal was generated after the beating of the first order modulated bands at the photodiodes (PD, Finisar XPDV2320R) at BS\#1 and BS\#3 points, and was directly monitored by the RF spectrum analyzer (RFSA) (Agilent N9020A MXA).

\section{Multiple RF signal generation and SSMF-FSO optical distribution}

In the following, five different scenarios with multiple RF signal generation for the ODN combining SSMF and FSO links were tested (see detailed in Table 1) and compared with the reference optical back to back (OB2B) link (denoted as scenario A). Fig. 2(a) and 2(b) show the measured optical spectra at both AWG active output ports \#1 and \#3 with suppressed carrier signal transmission.

Table 1. Definition of experimental scenarios

\begin{tabular}{|l|l|}
\hline Scenarios & Optical Distribution Network \\
\hline $\mathrm{A}$ & OB2B \\
\hline $\mathrm{B}$ & $10 \mathrm{~km}$ SSMF link \\
\hline $\mathrm{C}$ & $25 \mathrm{~km}$ SSMF link \\
\hline $\mathrm{D}$ & $70 \mathrm{~cm}$ FSO link \\
\hline $\mathrm{E}$ & $10 \mathrm{~km}$ SSMF $+70 \mathrm{~cm}$ FSO \\
\hline $\mathrm{F}$ & $\begin{array}{l}10 \mathrm{~km} \text { SSMF }+70 \mathrm{~cm} \text { FSO } \\
\text { (with post-detection RF amplification) }\end{array}$ \\
\hline
\end{tabular}

The carrier suppression level between sidebands and the optical carrier was measured as 20 $\mathrm{dB}$ at $7 \mathrm{GHz}$ for port \#1 and larger than $30 \mathrm{~dB}$ at $12 \mathrm{GHz}$ for port \#3. Moreover, next harmonic sidebands show power levels below $35 \mathrm{~dB}$ with respect to the fundamental sidebands. The spectra measured at output port \#1 (Fig. 2 (a)) and \#3 (Fig. 2 (b)) show the residual power from another AWG port with differences of $-33 \mathrm{~dB}$ and $-42 \mathrm{~dB}$, respectively. Note that nominal AWG crosstalk was $30 \mathrm{~dB}$. The ODN insertion losses were determined as $3.2 \mathrm{~dB}, 5.6 \mathrm{~dB}$, $4.6 \mathrm{~dB}$ and $7.8 \mathrm{~dB}$ for scenarios B, C, D and E defined in Table 1, respectively.

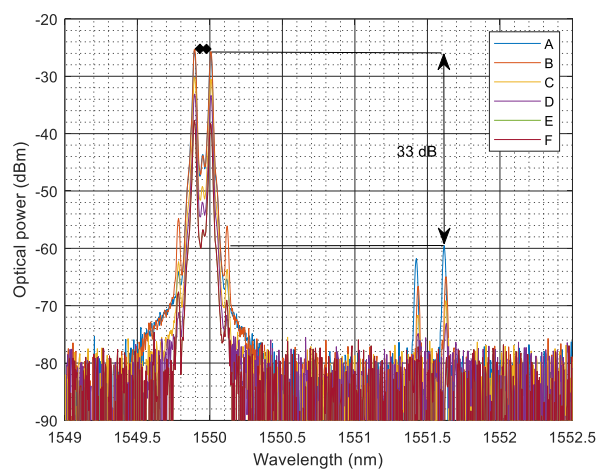

(a)

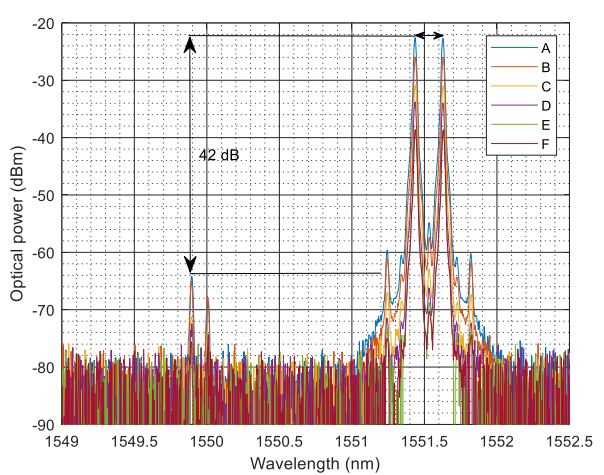

(b)

Fig. 2. Measured optical spectra at the photodetector input (RBW $=0.02 \mathrm{~nm})$ : (a) Port $\# 1: \lambda_{1}=$ $1549.90 \mathrm{~nm}, \mathrm{f}_{\mathrm{RF} 1}=7 \mathrm{GHz}$, (b) Port $\# 3: \lambda_{2}=1551,52 \mathrm{~nm}, \mathrm{f}_{\mathrm{RF} 2}=12 \mathrm{GHz}$.

After a direct photodetection, two signals were generated at $14 \mathrm{GHz}$ and $24 \mathrm{GHz}$. The RF spectra for both frequencies are depicted in Fig. 3(b) and Fig. 3(c), respectively. The spectral linewidths of the generated RF carriers were measured as $60 \mathrm{kHz}$ in all scenarios using a resolution bandwidth (RBW) of $51 \mathrm{kHz}$. The RF power levels for OB2B system were 
$-41.1 \mathrm{dBm}$ at both frequencies $14 \mathrm{GHz}$ and $24 \mathrm{GHz}$, measured by an RFSA with a RBW of $91 \mathrm{kHz}$. Fig. 3(a) shows the RF received power for a particular scenario A-D for both $14 \mathrm{GHz}$ and $24 \mathrm{GHz}$ in order to compare the performance for different radio frequencies. Whereas the received $\mathrm{RF}$ powers at $14 \mathrm{GHz}$ and $24 \mathrm{GHz}$ were comparable for the scenarios $\mathrm{A}, \mathrm{B}, \mathrm{C}, \mathrm{D}$ and E, a $6 \mathrm{~dB}$ difference was observed between RF power values measured at $14 \mathrm{GHz}$ and $24 \mathrm{GHz}$ in scenario $\mathrm{F}$ due to the frequency response of the amplifier (SHF Communication Technologies AG, SHF 810) and adaptors.

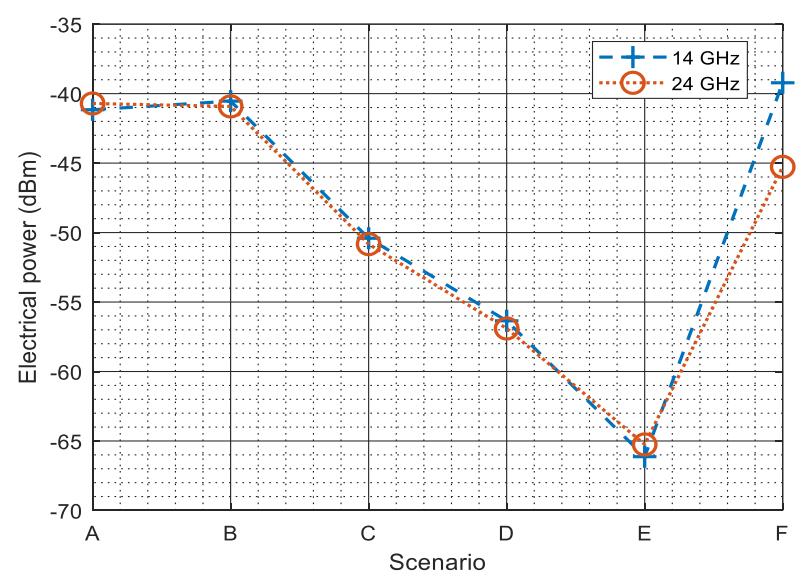

(a)

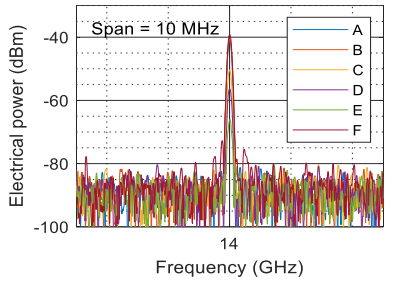

(b)

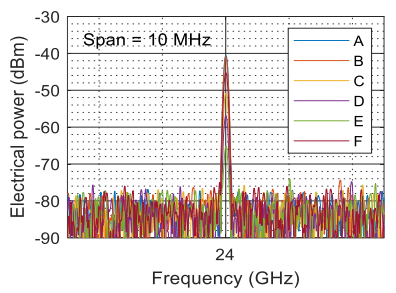

(c)

Fig. 3. (a) RF power level measured at different scenarios, (b) RF power spectrum at $\mathrm{f}_{\mathrm{RF} 1}=$ $14 \mathrm{GHz}$ (carried by $\lambda_{1}=1549.90 \mathrm{~nm}$ ) and (c) RF power spectrum at $\mathrm{f}_{\mathrm{RF} 2}=24 \mathrm{GHz}$ (carried by $\left.\lambda_{2}=1551.52 \mathrm{~nm}\right)$.

Since the signal phase noise is critical due to the degradation impact in digitally modulated communications systems, especially in up-converted $\mathrm{mmW}$ signals, we characterized it for all proposed system scenarios. Fig. 4 shows the phase noise level measurements at $14 \mathrm{GHz}$ and $24 \mathrm{GHz}$ at different scenarios. The lowest phase noise at $100 \mathrm{kHz}$ offset for OB2B was $-87.4 \mathrm{dBc} / \mathrm{Hz}$ and $-93.3 \mathrm{dBc} / \mathrm{Hz}$ at $14 \mathrm{GHz}$ and $24 \mathrm{GHz}$, respectively. Higher phase noise was detected for scenarios providing lower electrical signal power according to Fig. 3, as demonstrated e.g. in [26]. Nevertheless, our system provides $\mathrm{mmW}$ signal generation with phase noise level under $-75 \mathrm{dBc} / \mathrm{Hz}$ for scenarios $\mathrm{A}, \mathrm{B}, \mathrm{C}$ and $\mathrm{D}$, which is a reasonable level when compared e.g. with measurements in [27] $(-87.2 \mathrm{dBc}$ at $10 \mathrm{kHz}$ frequency offset) or with nominal phase noise of signal generator Agilent E8267C PSG $(-108 \mathrm{dBc} / \mathrm{Hz}$ from $10 \mathrm{GHz}$ to $20 \mathrm{GHz}$ at $20 \mathrm{kHz}$ frequency offset). Note that scenario E experienced a very low received RF power, which accordingly brings higher phase noise level, i.e. $-69 \mathrm{dBc} / \mathrm{Hz}$ at $24 \mathrm{GHz}$. However, it was decreased down to $-87 \mathrm{dBc} / \mathrm{Hz}$ for both selected frequencies when post-detection RF amplification was applied (scenario F), as can be also shown in the inset of Fig. 4. 


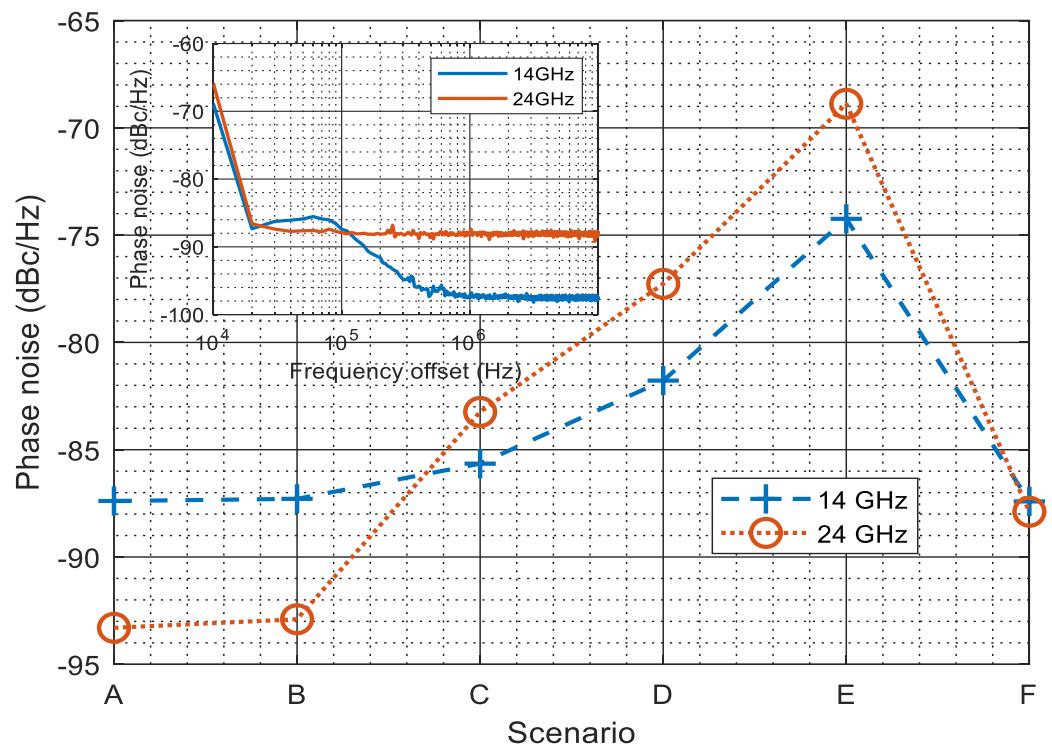

Fig. 4. Phase noise level ( $\mathrm{RBW}=10 \mathrm{kHz})$ measured at different scenarios $(100 \mathrm{kHz}$ offset). Inset: spectral phase noise measurement after transmission over scenario $\mathrm{F}$ for channel $1\left(\lambda_{1}=\right.$ $\left.1549.90 \mathrm{~nm}, \mathrm{f}_{\mathrm{RF} 1}=14 \mathrm{GHz}\right)$ and channel $3\left(\lambda_{2}=1551.52 \mathrm{~nm}, \mathrm{f}_{\mathrm{RF} 2}=24 \mathrm{GHz}\right)$.

\section{Photonic $\mathrm{mmW}$ signal generation and reconfigurable signal distribution}

In the next step, five optical channels were used to generate and carry signals at different frequencies in the mmW band through the hybrid SSMF and FSO distribution network to dynamically feed different BSs with particular frequencies. In this case, we employed an optical switch (OS, SW8x3-9N-FC/PC) and a cyclical 18x18 ports AWG (A0818GPMFS-B203A) as depicted in Fig. 5 with $7.9 \mathrm{~dB}$ of total insertion losses. DFB lasers emitting at $1547.47 \mathrm{~nm}$, $1548.25 \mathrm{~nm}, 1549.10 \mathrm{~nm}, 1549.85 \mathrm{~nm}$ and $1550.70 \mathrm{~nm}$ were modulated by five MZMs similar to those employed in section 2 at $20 \mathrm{GHz}, 15 \mathrm{GHz}, 12.5 \mathrm{GHz}, 10 \mathrm{GHz}$ and $7.5 \mathrm{GHz}$ in order to generate RF-mmW signals at $40 \mathrm{GHz}, 30 \mathrm{GHz}, 25 \mathrm{GHz}, 20 \mathrm{GHz}$ and $15 \mathrm{GHz}$, respectively. As an example, the inset in Fig. 5 illustrates the measured optical spectrum of the multiplexed signal when the channels at $1547.47 \mathrm{~nm}$ and $1548.25 \mathrm{~nm}$ were modulated by the $\mathrm{RF}$ corresponding signals. 


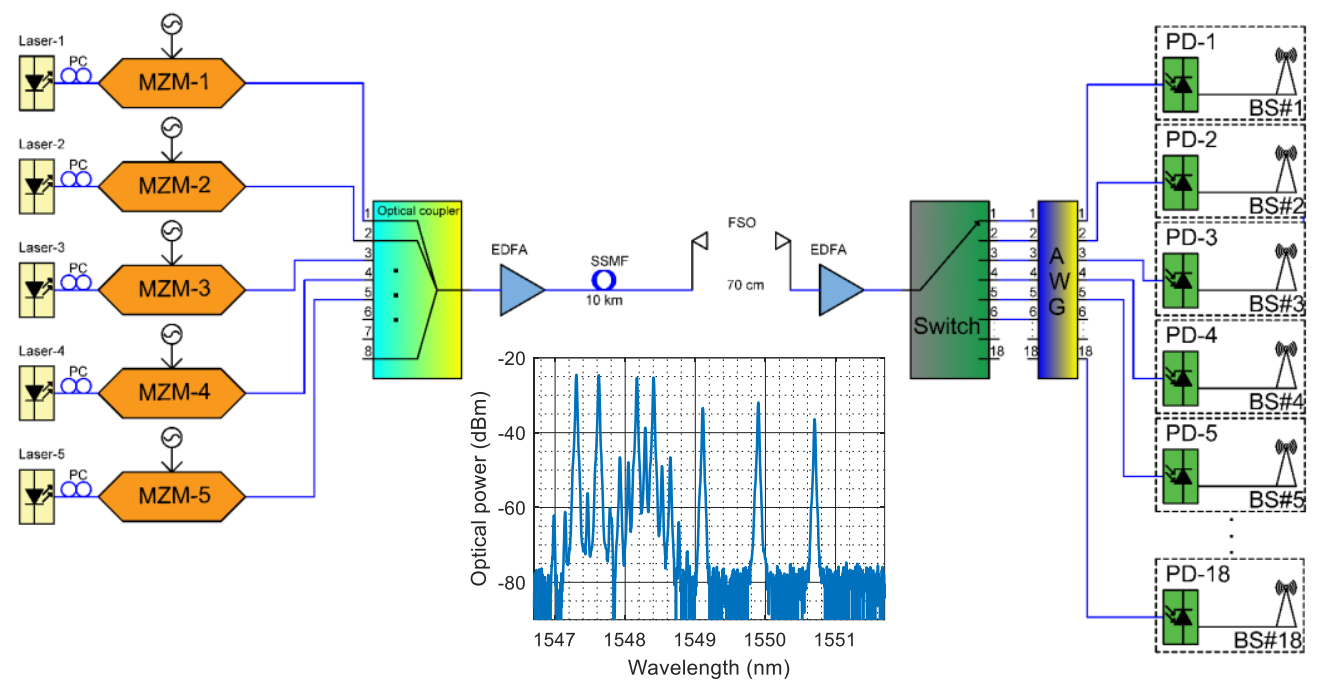

Fig. 5. Experimental setup of photonic multiple $\mathrm{mmW}$ signal generation and reconfigurable signal distribution. Inset: optical spectrum of five multiplexed channels, with Laser-1 and 2 emitting channels modulated with 40 and $35 \mathrm{GHz}$, respectively.

Fig. 6(a) shows the optical spectra just prior to the photodetection, whereas Fig. 6(b) depicts the electrical spectrum (measured by Agilent N4373C) at a particular receiver when the signal was launched through port \#2 in the AWG after the $10 \mathrm{~km} \mathrm{SSMF}$ and $70 \mathrm{~cm}$ FSO channel. Also shown are the carrier suppressed and modulated sidebands at different channels after AWG signal routing with a crosstalk level better than $-28 \mathrm{~dB}$. The optical carrier suppression with the minimal ratio of $17.81 \mathrm{~dB}$ was observed in all cases although BS\#2 exhibited weaker suppression due to differences in their frequency response and polarization sensitivity. Phase noise levels below $-85 \mathrm{dBc} / \mathrm{Hz}$ and linewidths under $60 \mathrm{kHz}$ were obtained at all BS\#s (Fig. 6 (b)). Then, the mmW signal, with minimal RF power of $-40 \mathrm{dBm}$, can be afterward used for further radiation in $\mathrm{BS} \# 1$ to $\mathrm{BS} \# 5$. 


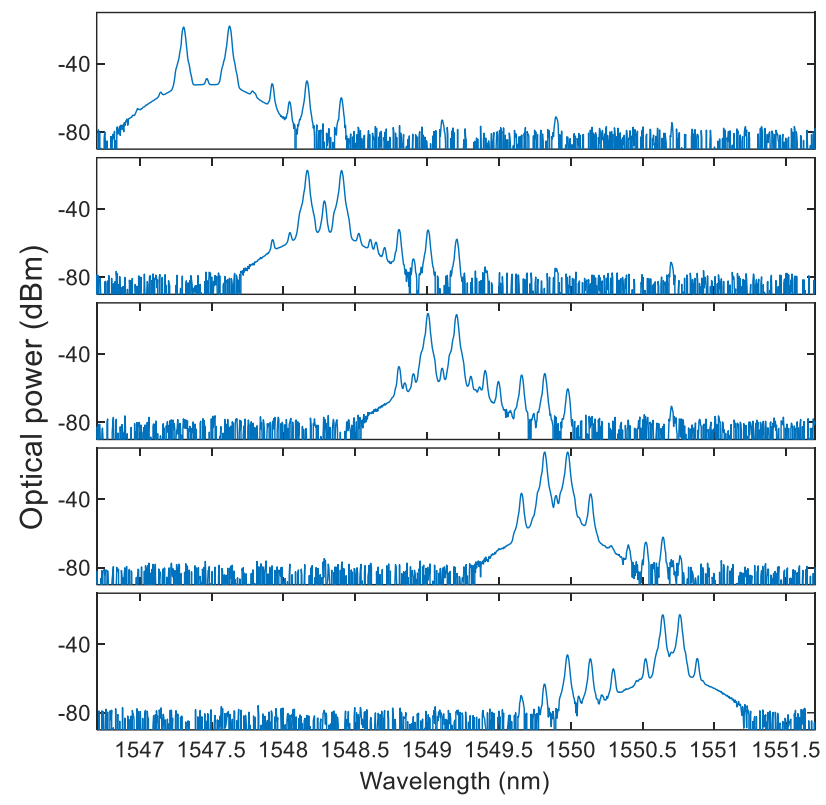

(a)

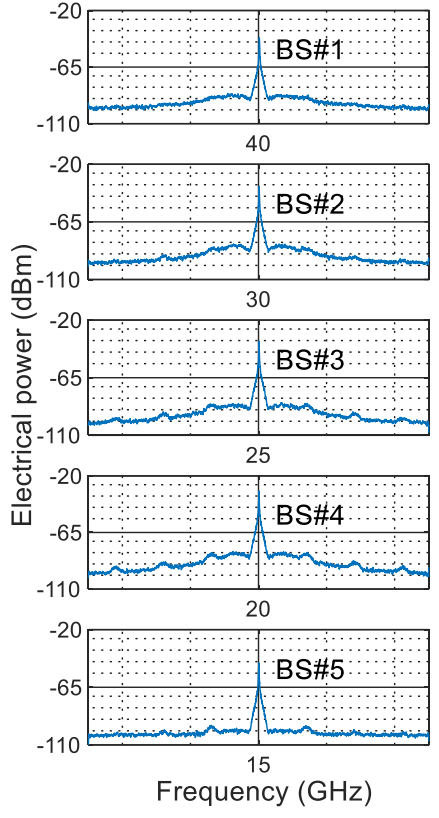

(b)

Fig. 6. Measurements at different BSs after distribution over the hybrid SSMF and FSO network when the signal is launched into AWG through input port \#2: (a) Optical spectra prior to photodetection, (b) Electrical spectra.

Fig. 7(a) shows every wavelength carrying the particular mmW signal and its corresponding output (BS\#) for different input ports where the examples inside the dashed box correspond to the ones depicted in Fig. 7(b). It shows the electrical spectra of signals recovered at different BSs when the optical switch was configured to launch the optical signal into a given input port of the AWG after distribution over the SSMF/FSO hybrid network. Depending on the configuration of the OS, the input port at the AWG was different and, after demultiplexing, a variable optical channel carrying a particular $\mathrm{mmW}$ signal was delivered to the BS for dynamic $\mathrm{RF}$ band reassignment and also for service provisioning. For example, we can observe that a $40 \mathrm{GHz}$ signal, carried by $1547.47 \mathrm{~nm}$, was distributed to BS\#18 or e.g. BS\#15 when the signal was launched into input AWG port \#3 or \#6 (Fig. 7(b)), respectively. Similarly, signals were switched to different input ports and electrical signals at $15 \mathrm{GHz}, 20 \mathrm{GHz}, 25 \mathrm{GHz}$, $30 \mathrm{GHz}$ and $40 \mathrm{GHz}$, generated after direct detection, were correctly routed to particular BS, according to the cyclical response of the AWG, with $-40 \mathrm{dBm}$ electrical power and noise phase level below $-75 \mathrm{dBc} / \mathrm{Hz}$. Remote software-assisted control of the OS would allow implementing a reconfigurable hybrid network in order to provide future dynamic mobile fronthaul mobile networks with the highest performance, expandability and reachability. 


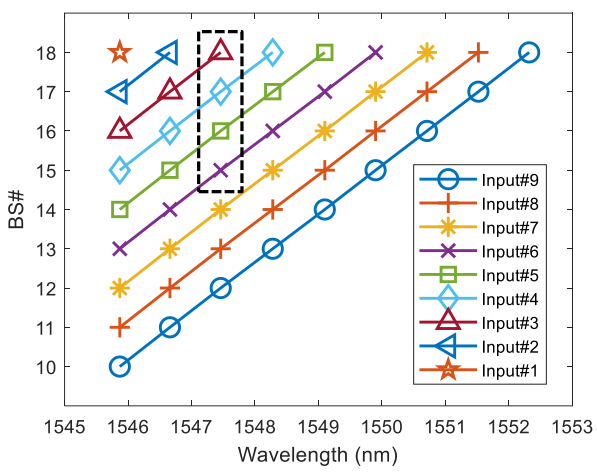

(a)

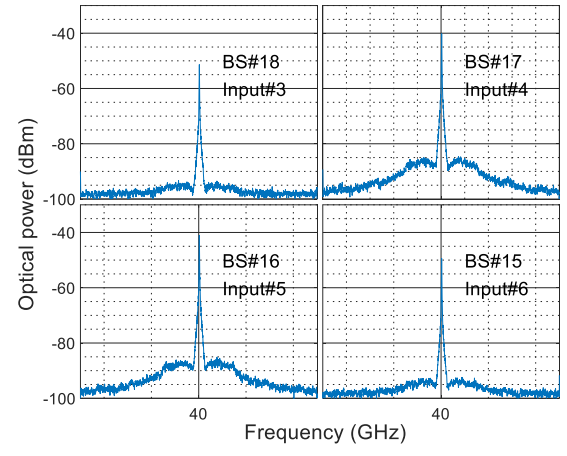

(b)

Fig. 7. (a) BS where the signal will be radiated versus optical carrier wavelengths when launched into different inputs of the AWG, (b) RF spectrum at $40 \mathrm{GHz}$ (carried by $1547.47 \mathrm{~nm}$ ) generated at different BSs according to the AWG input port (all graphs show a span of $1 \mathrm{MHz}$ ).

\section{Data transmission}

Finally, the proposed experimental setup with the photonically generated $\mathrm{mmW}$ signal has been tested for data transmission over hybrid link for usage in potential $5 \mathrm{G}$ network. A $40 \mathrm{GHz}$ optical $\mathrm{mmW}$ was generated at MZM-1 output by carrier suppression $\left(\mathrm{V}_{\mathrm{DC} 1}=6.5 \mathrm{~V}\right)$ according to the scheme depicted in Fig. 8(a). After EDFA amplification and polarization adjustment, the optical double-sideband signal with a suppressed carrier is brought to MZM-1D, which is biased at quadrature point $\left(\mathrm{V}_{\mathrm{DC}-1 \mathrm{D}}=3 \mathrm{~V}\right)$ and modulated by a 32-QAM signal with $2 \mathrm{GHz}$ signal data bandwidth centered at $2 \mathrm{GHz}$ with the pseudorandom binary sequence (PRBS) length of $\left(2^{9}-1\right)$ and bit rate of $10 \mathrm{~Gb} / \mathrm{s}$. The signal was generated by an arbitrary waveform generator (AWG, Tektronix AWG7122C) and amplified by a RF amplifier (Minicircuits, ZJL$4 \mathrm{HG}$ ). The modulated optical signal was amplified in another EDFA (20 dB gain Accelink EDFA-MW-BA) to compensate for MZM-1D insertion loss and launched in the hybrid link.

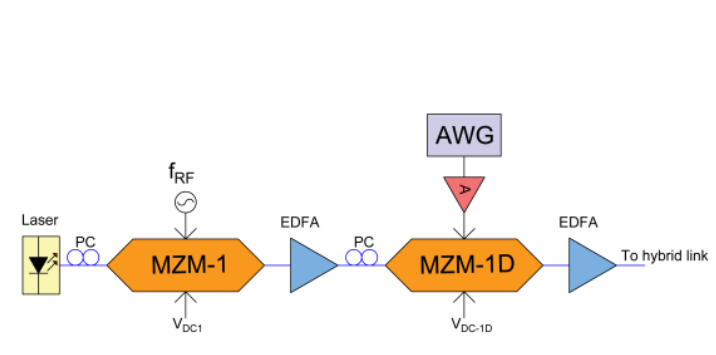

(a)

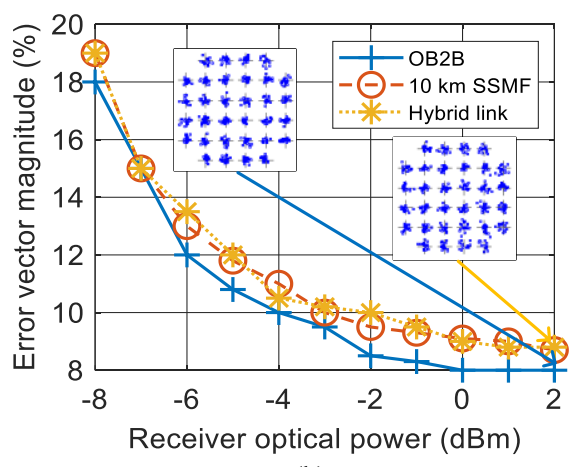

(b)

Fig. 8. (a) Schematic for photonically generated $\mathrm{mmW}$ signals and data transmission, (b) Measured error vector magnitude (EVM) curves of the $10 \mathrm{Gbps}$ over $40 \mathrm{GHz} \mathrm{mmW}$ data signal for OB2B and hybrid links (10 km SSMF and FSO). Insets: constellations of the recovered 32QAM signals (received optical power of $2 \mathrm{dBm}$ ).

The error vector magnitude (EVM) of $10 \mathrm{~Gb} / \mathrm{s}$ 32-QAM signal carried by $40 \mathrm{GHz} \mathrm{mmW}$ at the wavelength of $1547.47 \mathrm{~nm}$ was measured by a digital phosphor oscilloscope (DPO) (Tektronix DPO72004C) for different scenarios against the received optical power and plotted 
in Fig. 8(b). For optical power at the receiver up to $-6 \mathrm{dBm}$, the OB2B EVM is below $12 \%$, which is the EVM limit to achieve a bit error rate lower than the $3.8 \cdot 10^{-3}$ hard decoding FEC threshold for 32-QAM [28]. The fiber and hybrid link scenarios both impose a power penalty of only $1 \mathrm{~dB}$, principally due to the fiber dispersion and increased insertion losses. In addition, the difference between the $10 \mathrm{~km}$ SSMF link and the combined link with FSO under clear atmospheric conditions is negligible for $10 \mathrm{~Gb} / \mathrm{s}$ transmission. Note that the lowest obtained EVM is $8 \%$ and $8.5 \%$ for OB2B scenario and hybrid link, respectively, with $2 \mathrm{dBm}$ of received optical power.

\section{Conclusions}

In this paper, we proposed and demonstrated a simple and flexible scheme for the generation of multiple mmW signals using MZM biased in zero transmission point based on WDM technology with reconfigurable distribution to different base stations suitable for small cell network architectures. Furthermore, the transmission over hybrid optical fiber and FSO links has been experimentally evaluated. Optical carrier suppression at the MZM has been employed to generate doubling radio input frequency signals in the $15-40 \mathrm{GHz}$ range with the phase noise level as low as $-87 \mathrm{dBc} / \mathrm{Hz}$ without the need for additional optical filters. Moreover, multiple signal distribution over the hybrid optical network including optical fiber and FSO link has been demonstrated and high-quality RF signals have been generated at the BSs. It has been shown that AWG can assist and remotely switch reconfiguration at the RN to demonstrate the high potential of the proposed system for dynamic $5 \mathrm{G}$ networks deployment. Finally, $10 \mathrm{~Gb} / \mathrm{s} 32-\mathrm{Q} A M$ data signal has been successfully transmitted over the proposed system with up to $40 \mathrm{GHz} \mathrm{mmW}$ signal along the hybrid link with a minimum EVM of $8.5 \%$.

\section{Funding}

This work has been funded by the Research Excellence Award Programme GVA PROMETEO 2017/103 Future Microwave Photonics Technologies and Applications and MEYS INTERCOST project LTC18008 (within COST action CA 16220).

\section{References}

1. R. Waterhouse and D. Novack, "Realizing 5G: microwave photonics for 5G mobile wireless systems," IEEE Microwave Magazine, vol. 16, no. 8, pp. 84-92, Sept. 2015.

2. A. Hirata, H. Takahashi, R. Yamaguchi, T. Kosugi, K. Murata, T. Nagatsuma, N. Kukutsu and Y. Kado, "Transmission characteristics of $120 \mathrm{GHz}$-band wireless link using radio-on-fiber technologies," Journal of Lightwave Technology, vol. 26, no. 15, pp. 2338-2344, Aug. 2008.

3. C. H. Lee, Microwave Photonic, 2nd ed., Taylor \& Francis, 2013.

4. A. Checko, H. L. Christiansen, Y. Yan, L. Scolari, G. Kardaras, M. S. Berger and L. Dittmann, "Cloud RAN for mobile networks - A technology overview," IEEE Communications Surveys \& Tutorials, vol. 17, no. 1, pp. 405-426, 2015.

5. P. T. Dat, A. Kanno and T. Kawanish, "Radio-on-radio-over-fiber: efficient fronthauling for small cells and moving cells," IEEE Wireless Communications, vol. 22, no. 5, pp. 67-75, 27 Oct. 2015.

6. C. Lim, A. Nirmalathas, M. Bakaul, P. Gamage, K.-L. Lee, Y. Yang, D. Novak and R. Waterhouse, "Fiberwireless networks and subsystem technologies," Journal of Lightwave Technology, vol. 28, no. 4, pp. 390-405, Feb. 2010

7. J. Beas, G. Castanon, I. Aldaya, A. Aragon-Zavala and G. Campuzano, "Millimeter-wave frequency radio over fiber systems: a survey," IEEE Communications Surveys \& Tutorials, vol. 15, no. 4, pp. 1593-1619, 2013.

8. J. Yao, "Microwave photonics," Journal of Lightwave Technology, vol. 27, no. 3, pp. 314-335, Feb. 2009.

9. Y. Doi, S. Fukushima, T. Ohno and K. Yoshino, "Frequency stabilization of millimeter-wave subcarrier using laser heterodyne source and optical delay line," IEEE Photonics Technology Letters, vol. 13, no. 9, pp. 10021004, Sept. 2001.

10. A. Hurtado, I. D. Henning, M. J. Adams and L. F. Lester, "Generation of tunable millimeter-wave and THz signals with an optically injected quantum dot distributed feedback laser," IEEE Photonics Journal, vol. 5, no. 4, Aug. 2013. 
11. J. Liu, H.-C. Chien, S.-H. Fan, B. Chen, J. Yu, S. He and G.-K. Chang, "Efficient optical millimeter-wave generation using a frequency-tripling fabry-pérot laser with sideband injection and synchronization," IEEE Photonics Technology Letters, vol. 23, no. 18, pp. 1325-1327, Sept. 2011.

12. T. Nakasyotani, H. Toda, T. Kuri and K. Kitayama, "Wavelength-division-multiplexed Millimeter-waveband radio-on-fiber system using a supercontinuum light source," Journal of Lightwave Technology, vol. 24, no. 1, pp. 404-410, Jan. 2006.

13. L. Xu, C. Li, S. M. G. Lo and H. K. Tsang, "Millimeter wave generation using four wave mixing in silicon waveguide," OECC 2010 Technical Digest, pp. 860-861, Jul. 2010.

14. Y.-K. Seo, C.-S. Choi and W.-Y. Choi, "All-optical signal up-conversion for radio-on-fiber applications using cross-gain modulation in semiconductor optical amplifiers," IEEE Photonics Technology Letters, vol. 14, no. 10, pp. 1448-1450, Oct. 2002.

15. T. Kanesa, F. Maskuriy, M. Hafiz, R. Mohamad, S. M. Mitani, H. M. Hizan, A. I. A. Rahim, P. A. Haigh, S. Rajbhandari and G. Chang, "Dual pump brillouin laser for RoF millimeterwave carrier generation with tunable resolution," TENCON 2015 - 2015 IEEE Region 10 Conference, pp. 1-6, Nov. 2015.

16. G. Qi, J. Yao, J. Seregelyi, S. Paquet and C. Belisle, "Generation and Distribution of a Wide-Band Continuously Tunable Millimeter-Wave Signal With an Optical External Modulation Technique," IEEE Transactions on Microwave Theory and Techniques, vol. 53, no. 10, pp. 3090-3097, Oct. 2015.

17. H. Zhang, C. Lin, S. Xie, K. Zhang, X. Wu and Z. Dong, "A novel radio-over-fiber system based on carrier suppressed frequency eightfold millimeter wave generation," IEEE Photonics Journal, vol. 9, no. 5, pp. 1-6, Oct. 2017.

18. L. Zhang, M. Zhu, C. Ye, S.-H. Fan, C. Liu, X. Hu, P. Cao, Q. Chang, Y. Su and G.-K. Chang, "Generation and transmission of multiband and multi-gigabit $60-\mathrm{GHz}$ MMW signals in an RoF system with frequency quintupling technique," Optics Express, vol. 21, no. 8, pp. 9899-9905, Apr. 2013.

19. Z. Zhao, Z. Zhang, T. Jun, L. Yu and J. Liu, " $200 \mathrm{~Gb} / \mathrm{s}$ FSO WDM communication system empowered by multiwavelength directly modulated TOSA for 5G wireless networks," IEEE Photonics Journal, vol. 10, no. 4, pp. 1-8, Aug. 2018.

20. J. Zhang, J. Wang, Y. Xu, M. Xu, F. Lu, L. Heng, J. Yu and G.-k. Chang, "Fiber-wireless integrated mobile backhaul network based on a hybrid millimeter-wave and free-space-optics architecture with an adaptive diversity combining technique," Optics Letters, vol. 41, no. 9, pp. 1909-1912, 2016.

21. R. Zhang, F. Lu, M. Xu, S. Liu, P.-C. Peng, S. Shen, J. He, H. J. Cho, Q. Zhou, S. Yao and G.-K. Chang, “An ultra-reliable MMW/FSO A-RoF system based on coordinated mapping and combining technique for 5G and beyond mobile fronthaul," An Ultra-Reliable MMW/FSO A-RoF System Based on Coordinated Mapping and Combining Technique for $5 G$ and Beyond Mobile Fronthaul, vol. 36, no. 20, pp. 4952-4959, 2018.

22. J. Bohata, M. Komanec, J. Spáčil, Z. Ghassemlooy, S. Zvánovec and R. Slavík, "24-26 GHz radio over fiber and free space optics for 5G systems," Optics Letters, vol. 43, no. 5, pp. 1035-1038, Mar. 2018.

23. C.-T. Lin, J. Chen, S.-P. Dai, P.-C. Peng and S. Chi, "Impact of nonlinear transfer function and imperfect splitting ratio of MZM on optical up-conversion employing double sideband with carrier suppression modulation," Journal of Lightwave Technology, vol. 26, no. 15, pp. 2449-2459, 2008.

24. G. Ning, P. Shum and J. Zhou, "Dispersion effect and compensation in optical-carrier-suppressed modulation transport systems," Journal of the Optical Society of America A, vol. 24, no. 11, pp. 3432-3436, 2007.

25. M. Uysal, C. Capsoni, Z. Ghassemlooy, A. Boucouvalas and E. Udvary, Optical WirelessCommunications - An Emerging Technology, Springer, 2016.

26. G. Qi, J. Yao, J. Seregelyi, S. Paquet, C. Belisle, X. Zhang, K. Wu and R. Kashyap, "Phase-noise analysis of optically generated millimeter-wave signals with external optical modulation techniques," Journal of Lightwave Technology, vol. 24, no. 12, pp. 4861-4875, 2006.

27. X. Lin, C. Li, C. W. Chow and H. K. Tsang, "Optical mm-wave signal generation by frequency quadrupling using an optical modulator and a silicon microresonator filter," IEEE Photonics Technology Letters, vol. 21, no. 4, pp. 209-211, Feb. 2009.

28. International Telecommunication Union, "Forward error correction for high bit-rate DWDM submarine systems," ITU-T Recommendation G.975.1, 2004. 